\title{
The Endogenous Modulation of IOR is Nasal-Temporal Asymmetric
}

\author{
Andrea Berger and Avishai Henik \\ Ben-Gurion University of the Negev and The Zlotowski Center for Neuroscience, Israel
}

\begin{abstract}
- Inhibition of return (IOR) refers to a reflexive mechanism mediated by phylogenetically primitive extrageniculate visuomotor pathways, which apparently serves to favor novel spatial locations by inhibiting those recently sampled. We demonstrate an asymmetry between temporal and nasal hemifields in the strategic modulation of IOR by endogenously controlled attention. Exogenous and endogenous precues were manipulated independently on each trial such that precues to initiate endogenous spatial orienting were presented after IOR had been activated by exogenous visual signals. Both types of precues manifested their characteristic effects on reaction time
\end{abstract}

(RT) to detect subsequent targets: facilitation by endogenous precues, and IOR by exogenous precues. Under monocular viewing, an asymmetric interaction between these two mechanisms was observed. While endogenous allocation of attention to the nasal hemifield reduced IOR, no endogenous modulation of IOR was present in the temporal hemifield where the effects of the two types of precues were independent. These observations suggest a framework for understanding the neurobiology of automaticity and controlfrom an evolutionary perspective.

\section{INTRODUCTION}

The visual system provides a clear example and a good prototype for studying the relation between ancient functions and higher processes, since in the mammal's brain there are actually two visual pathways. The ancient pathway in terms of phylogenetic development is subcortical and mainly involves projections from the retina to the superior colliculus (SC) of the midbrain. The newer pathway projects from the retina to the lateral geniculate body (LGB) and then to the striate cortex. One of the main principles of ontogenetic development is that subcortical mechanisms tend to develop prior to cortical mechanisms. These developmental courses lead to the following intriguing questions: What happens to the functions of the older visual system during the phylogenetic and ontogenetic processes? How are the primitive reflexes of the older visual pathway adapted by cortical systems to aid goal-directed behavior? This paper will focus on one of these primitive mechanisms, called inhibition of return (IOR). We examined the ability to strategically modulate IOR and used this modulation as a framework to provide insight into the neurobiology of automaticity and control from an evolutionary perspective.

\section{Inhibition of Return}

In order to survive, it is critical for organisms to react quickly and appropriately to events in their surround-

ings. Stimuli appearing in the peripheral-visual field have been shown to reflexively capture attention, improving visual performance in that spatial location (Jonides, 1981; Posner, 1980). Once attention is allocated to a given location, it is important that it is not captured there indefinitely, so that the system maintains its ability to scan other spatial locations. It has been suggested that IOR is a mechanism whereby the attentional system favors novel spatial locations by inhibiting alreadyscanned ones. This mechanism was first reported by Posner and Cohen (1984). In the experimental paradigm known as Posner's spatial-cueing task (Posner, 1980), a constant display consists of three unfilled squares, one in the center of the screen and the other two, $8^{\circ}$ right and left of center. An exogenous precue is created by making one of the squares brighter, each square being highlighted an equal number of times. The target stimulus, an asterisk, appears inside one of the two peripheral squares (50\% of trials in each), so that three types of trials could be distinguished: "valid" (target stimulus in the precued location), "invalid" (target stimulus in the opposite location), and "neutral" (target stimulus preceded by a central precue). Many studies have shown that when the target is presented shortly after the precue, subjects respond faster to a target stimulus presented in a cued location (valid cue trial), as compared to their response to a target at an uncued location (invalid cue trial) (Berger, Dori, \& Henik, 1999; Jonides, 1981; Muller \& Humphreys, 1991; Reuter-Lorenz \& 
Frendrich, 1992). IOR means reversal of this effect. It commonly happens when the interval between cue onset and target onset (SOA) is longer than $300 \mathrm{msec}$. Response to the target stimulus becomes slower when it follows a valid cue as compared to an invalid cue (Maylor, 1985; Maylor \& Hockey, 1985; Maylor \& Hockey, 1987; Posner \& Cohen, 1984).

There is accumulating evidence that suggests a strong relation between IOR and the extrageniculate visual pathways, and more specifically, the SC. One line of evidence is based on the temporal-nasal asymmetry. The extrageniculate visual pathway, unlike the geniculo-striate pathway, seems to have more crossed fibers (from the medial hemiretina [temporal hemifield] of the contralateral eye) than uncrossed fibers (from the lateral hemiretina [nasal hemifield] of the ipsilateral eye). This asymmetry is well established in cats, where the asymmetry rate is $80-20$ (Sherman, 1974). In primates, there is conflicting evidence regarding the projections from the retina to the SC. While Hubel, LeVay, and Wiesel (1975), Wilson and Toyne (1970), and Pollack and Hickey (1979) reported such asymmetry, Williams, Azzopardi, and Cowey (1995) recently found none. In humans, the exact proportion of crossed and uncrossed fibers projecting to the SC is not known. The behavioral evidence of such an asymmetry, however, seems to be very strong and consistent, and asymmetry is considered to be a useful marker for the extrageniculate visual pathway, since in the geniculo-striate pathway there is no temporal-nasal asymmetry. Temporal hemifield oculomotor advantage is found in newborns and adults (Mauer \& Lewis 1998; Posner \& Cohen, 1980). Newborns are especially interesting since their vision is "collicular" and considered to be mostly mediated by the retino-tectal pathway (since their geniculo-striate pathway is not yet fully developed). They show a strong bias to saccade to signals in the temporal-visual hemifield. In adults, there is still a bias to make a saccade toward the temporal hemifield even under conditions of bilateral, simultaneous stimulation. Based on this asymmetry, Rafal, Calabresi, Brennan, and Sciolto (1989) showed larger IOR in the temporal than in the nasal hemifield.

A second line of evidence concerning the connection between IOR and SC involves the examination of progressive supranuclear palsy (PSP) patients. PSP is a degenerative disease affecting the SC. These patients lose the ability to make voluntary saccadic eye movements, with vertical saccades usually lost before the horizontal saccades. Posner, Rafal, Choate, and Vaughn (1985) studied IOR in patients suffering from PSP. These patients were shown to have a deficit in IOR when covert orienting was demanded in the vertical dimension, while their IOR was preserved in the horizontal dimension.

A third line of evidence shows IOR in situations where the geniculo-striate, cortical-visual pathway is not func- tioning. This was shown in newborns (Simion, Valenza, Umilta, and Dalla-Baba, 1995) and hemianopic patients (Danziger, Fendrich, and Rafal, 1997).

Recently, we reported even more direct evidence (Sapir, Soroker, Berger, and Henik, 1999): A patient with a hemorrhage confined to the right SC showed normal IOR in the hemifields projecting to the intact SC, and showed no IOR in the hemifields projecting to the damaged SC. (See also Taylor and Klein (1998) for a comprehensive review of the IOR literature and the evidence regarding SC involvement in this effect.)

\section{Voluntary Modulation of IOR}

As we mentioned above, the primary question that we addressed was whether it is possible to strategically modulate a reflexive mechanism as IOR. More specifically, we asked what would happen if the subject voluntarily focuses attention on the exact location where IOR is developing. What would happen when there is a contradiction between the reflexive IOR process and a higher voluntary process?

We wanted to manipulate voluntary/endogenous attention at the same time that IOR was elicited. In the framework of Posner's paradigm, endogenous orienting is usually elicited by an arrow that points to the left or to the right and has predictive validity regarding the future location of the target. The orienting is based, in this case, on the symbolic meaning of the arrow and its predictive value; therefore, it is considered to be voluntary and controlled, in contrast to the automatic and reflexive fashion of the exogenous cue effect. Usually the arrow predicts correctly where the target is going to appear in $80 \%$ of the trials. In the neutral condition, a double-headed arrow is presented. When attention is summoned endogenously, usually no IOR is generated ${ }^{1}$ and subjects are able to maintain attention focused at the cued location, even at large SOAs.

\section{RESULTS}

\section{Experiment 1}

In our study, an endogenous cue, an arrow, and an exogenous cue, a brightening, were both presented in each trial (see Figure 1). The validity of each cue was manipulated independently. The endogenous cue appeared after IOR was already elicited by the exogenous cue, and it could summon attention to the same or opposite location.

For each subject, median reaction times (RT) were calculated for each experimental condition and a fourway repeated measures ANOVA (endogenous cue valid-

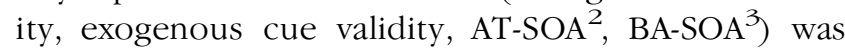
conducted (see Methods section for details regarding the design of the experiment).

The results showed that the characteristic effects of each cue were obtained. The main effect of the endo- 


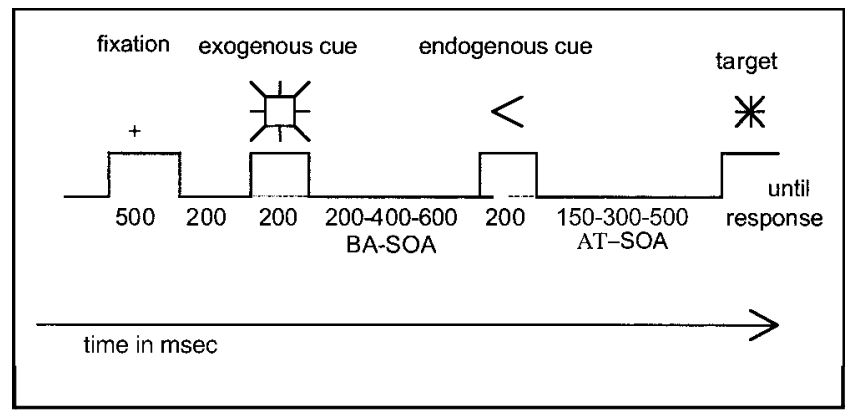

Figure 1. Order of events in a typical trial.

genous cue showed shorter RTs in the valid trials than in the invalid trials $(F(1,14)=86.5, M S E=1814, p<.001)$ (see Figure 2). The main effect of the exogenous cue showed the opposite pattern: RT was longer in the valid trials than in the invalid trials, or in other words, IOR was obtained $(F(1,14)=60.33, M S E=1378, p<.001)$ (see Figure 3).

IOR was larger at longer SOAs $(F(2,28)=5.4, M S E=$ $725, p<.01)$. Further analysis showed that the IOR was significant at all SOAs: $(F(1,14)=23.9, M S E=$ $1097, p<.001),(F(1,14)=10.13, M S E=1109$, $p<.01),(F(1,14)=85.9, M S E=623, p<.001)$, for 150 , 300 , and 500 msec, respectively. ${ }^{4}$

Regarding the IOR modulation: We found an interaction between the two cues $(F(1,14)=13.5, M S E=$ $717, p<.01$ ) (see Figure 4), showing larger IOR when the endogenous cue was invalid. Further analysis showed that the IOR was significant for both endogenous cue conditions. When the endogenous cue was valid, IOR size was $26 \mathrm{msec}(F(1,14)=21.2, M S E=$ $849, p<.001)$ and when it was invalid, IOR size was $34 \mathrm{msec}(F(1,14)=59.9, M S E=1246, p<.0001)$. It is important to notice that in the endogenously valid trials, IOR developed at the same location where the endogenous attention was focused. That means that the inhibition following the exogenous cue developed at the same location facilitated by the endogenous cue. Even in these conditions, attention could not prevent IOR.

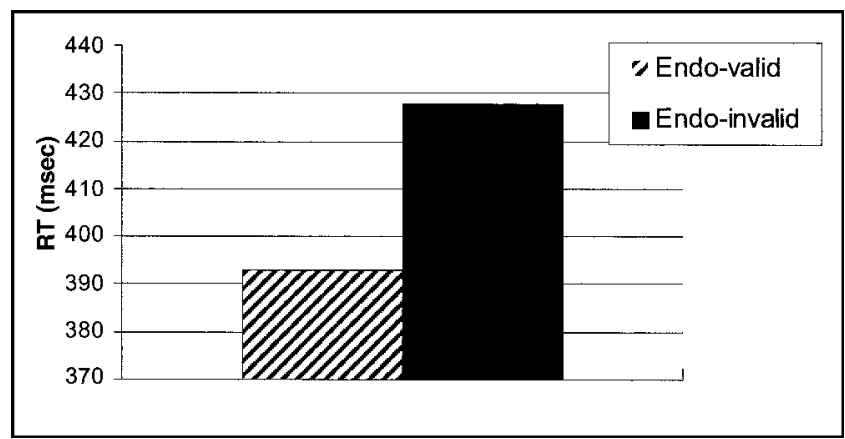

Figure 2. Endogenous effect in Experiment 1. Valid trials were faster that invalid trials.

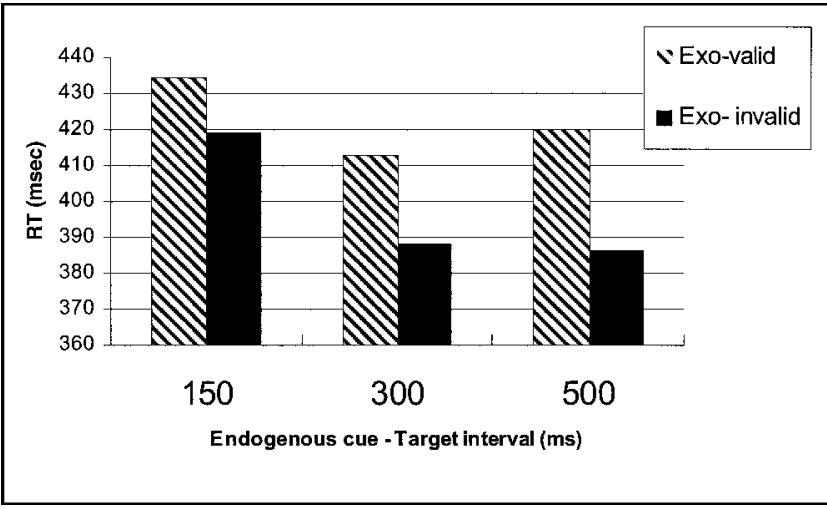

Figure 3. Interaction between the exogenous cue and SOA in Experiment 1. IOR was larger at longer SOAs and significant in all of them.

No higher interactions were found to be significant.

\section{Discussion}

In this experiment, we manipulated the endogenous and exogenous cues independently, presenting both types of cues in every trial. Attention could then be oriented endogenously toward the same or opposite location where IOR was developing.

An important aspect of the results that should be pointed out first is that in this experiment, all the intervals between the exogenous cue and the target were long. For this reason, we found IOR in the whole range that was tested.

Second, it is important to notice that the characteristic effects for each cue were found in conditions in which they were both acting at the same time, and sometimes even in contradicting directions. We suggest that this finding is consistent with the idea of two separate orienting mechanisms (Berger, Henik, Rafal, \& Egly, submitted; Briand \& Klein, 1987; Muller \& Humphreys, 1991; Reuter-Lorenz \& Frendrich, 1992). That means that the inhibition following the exogenous cue developed at the same location facilitated by the endogenous

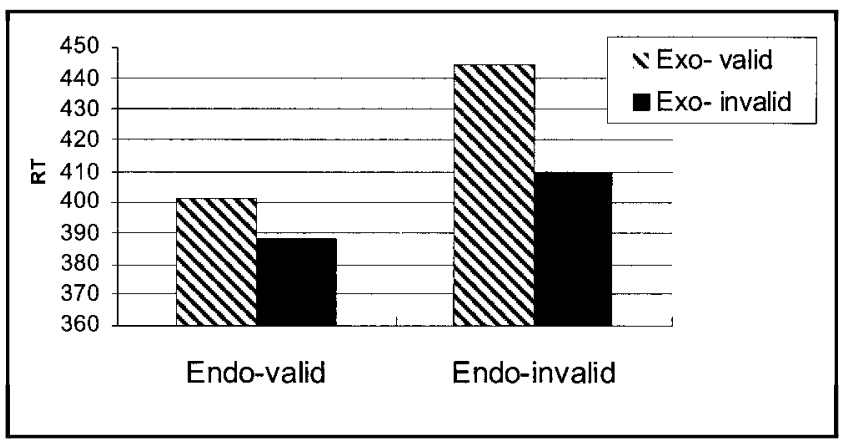

Figure 4. Interaction between the exogenous cue and the endogenous cue in Experiment 1. IOR was larger in the invalid endogenous condition. 


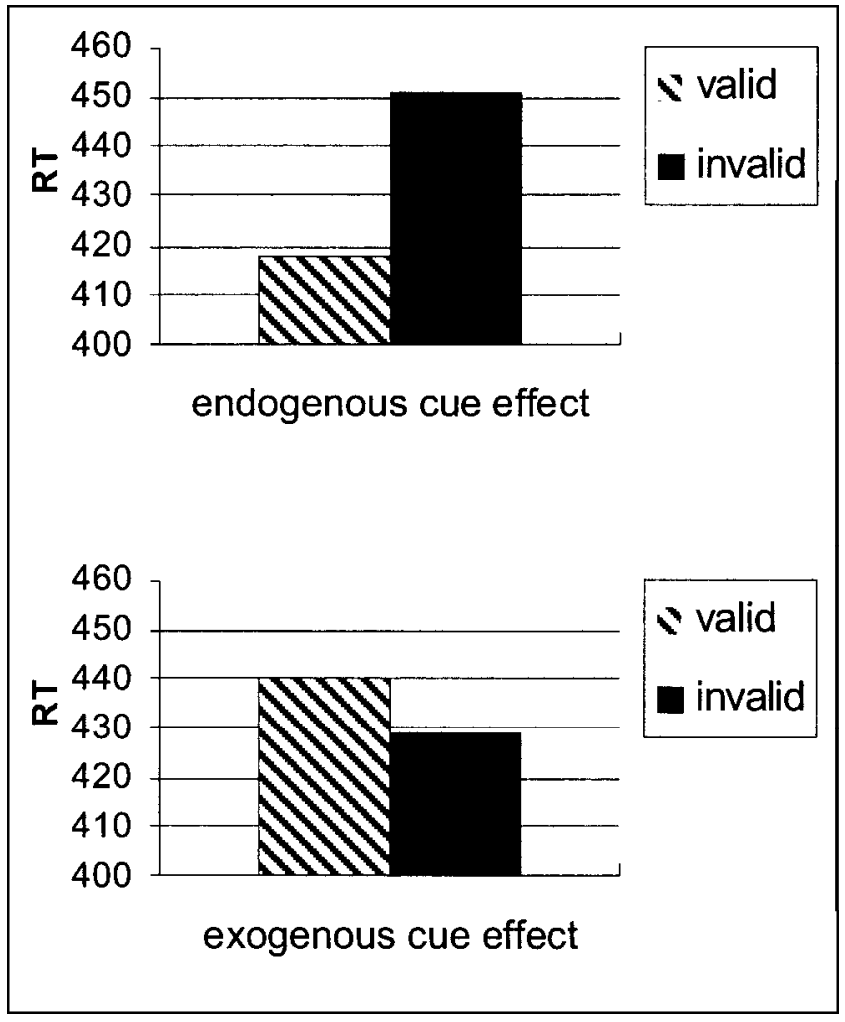

Figure 5. Endogenous and exogenous effects in the temporal hemifield in Experiment 2.

cue. Even in these conditions, attention could not prevent IOR. This finding is consistent with a recent report by Rafal and Henik (1994). In their study, IOR developed even when there was only one possible target position, and a strong incentive to maintain attention at that location.

The fact that the interaction between the cues' effects was not affected at all by the timing variables is also consistent with the interpretation of two separated mechanisms of attention. Yantis and Jonides (1990) found that endogenous orienting modulated the effect of an abrupt onset only when the endogenous cue preceded the abrupt onset by $200 \mathrm{msec}$. In the present experiment, the synchronization between the cues' onsets did not affect their interaction at all.

The present findings are also consistent with those of Kwak (1996). In the first experiment, he presented two cues in each trial, as we did, but simultaneously. After 700 or $1200 \mathrm{msec}$, a target was presented. Kwak found an interaction between the two cues, similar to the one obtained here. Namely, IOR was larger when the endogenous cue was invalid $(52 \mathrm{msec})$ than when the endogenous cue was valid $(36 \mathrm{msec})$. In the second experiment, he triggered endogenous and exogenous orienting with the same cue: an arrow presented peripherally. Independent effects of the two orienting mechanisms were found: endogenous facilitation and exogenous IOR.
We found an interaction between the two cues. This interaction could indicate one of two possibilities: (1) When the endogenous cue was invalid, the inhibition of the two cues resulted in a larger overall inhibition (IOR increased in this case); or (2) when the endogenous cue was valid, its facilitation counteracted IOR and resulted in a smaller IOR. Therefore, the next experiment was designed in order to further explore the relationship between endogenous orienting of attention and IOR.

\section{Experiment 2}

The idea of this experiment was to exploit the temporal-nasal asymmetry of the extrageniculate visual pathway (mentioned in the Introduction), in order to see whether the endogenous modulation of IOR is temporal-nasal asymmetric. According to the temporal-nasal asymmetry, it was predicted that IOR would be stronger and less susceptible to modulation in the temporal hemifield. We used the same design as in the previous experiment, but with monocular presentation.

In order to analyze the data across the two eyes, the trials were sorted according to hemifield and then, subsequently, the two blocks of data were combined. In this way, the temporal condition included temporal trials of both eyes, and the nasal condition included nasal trials of both eyes. For each subject, median RTs were calculated for each experimental condition. A four-way repeated measures ANOVA (endogenous cue validity, exogenous cue validity, SOA, hemifield) was conducted.

There were three main effects: endogenous cue $(F(1$, $22)=87.6, M S E=1659, p<.001) ;$ exogenous cue $(F(1,22)=19.7, M S E=1248, p<.001) ;$ and SOA $(F(2,44)=54.5, M S E=2076, p<.0001)$.

The interaction SOA $\times$ endogenous cue $\times$ exogenous cue was significant $(F(2,44)=4.06, M S E=$ $514, p<.05)$, but it was not analyzed further since it was included in the four-way interaction SOA $\times$ endogenous cue $\times$ exogenous cue $\times$ hemifield

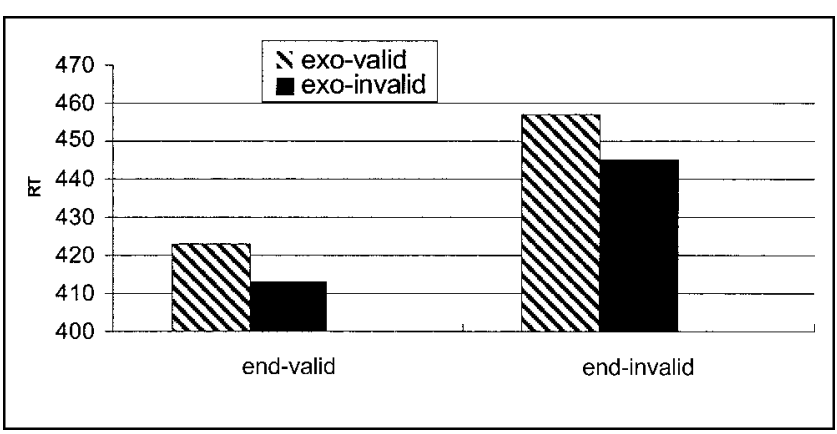

Figure 6. Experiment 2 . In the temporal hemifield, IOR was exactly the same regardless of where the attention was endogenously focused. 


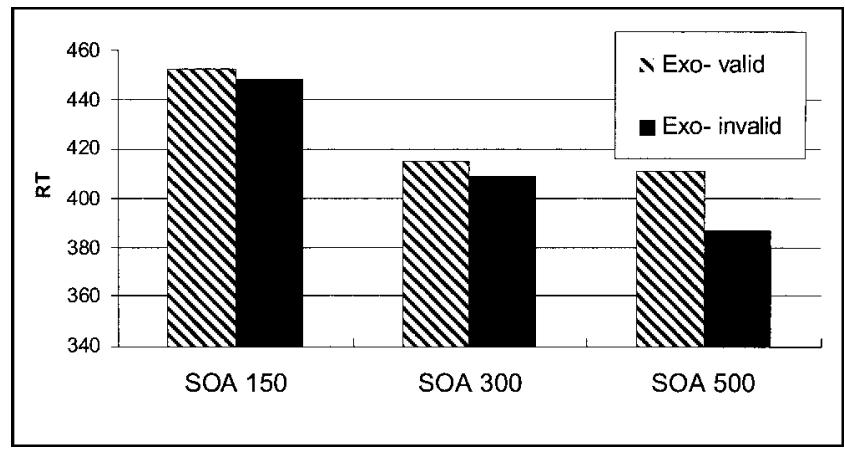

Figure 7. Experiment 2. Nasal hemifield. Interaction between exogenous cues and SOA in the endogenous valid condition. IOR found only at the long SOA.

that was significant $(F(2,44)=3.6, M S E=443, p<.05)$. Additional analysis was carried out separately for each hemifield, showing clear evidence of asymmetry between the temporal and nasal hemifields.

\section{Temporal Hemifield}

In the temporal hemifield, the results showed that the characteristic effects of each cue were obtained. The main effect of the endogenous cue showed shorter RTs in the valid trials than in the invalid trials $(F(1,22)=122.2, M S E=613, p<.0001)$. The main effect of the exogenous cue showed the opposite pattern: RT was longer in the valid trials than in the invalid trials, or in other words, IOR $(F(1,22)=$ 9.8, $M S E=842, p<.01$ ) (see both effects in Figure $5)$. The SOA main effect was also significant $(F(2,44)$ $=41.8, M S E=1,498, p<.0001)$.

No interactions were found in the temporal hemifield. As clearly illustrated in Figure 6, IOR was exactly the same no matter where attention was voluntarily focused. It seems that in this hemifield the reflexive IOR function could not be modulated at all.

\section{Nasal Hemifield}

In this hemifield, the simple main effects of SOA, exogenous cue, and endogenous cue were significant $((F(2,44)=47.7, M S E=1,065, p<.0001) ;(F(1,22)=$ 25.6, $M S E=673, p<.0001) ;$ and $(F(1,22)=53.8$, $M S E=1,308, p<.0001)$, respectively). In contrast to the picture found in the temporal hemifield, in the nasal hemifield there was a significant interaction between the endogenous and the exogenous cues $(F(2,44)=5.07, M S E=296, p<.05)$, and a significant three-way interaction between SOA and the two cues $(F(2,44)=7.9, M S E=452, p<.001)$.

Further analysis of the three-way interaction showed that when the endogenous cue was valid (meaning that IOR was expected to develop in the exact location where the subject was voluntarily focusing attention) there was an interaction between SOA and the exogenous cue $(F(2,44)=6.51, p<.01)$, and IOR showed up and was significant only at the longer SOA $(F(1,22)=$ 16.74, $p<.001$ ) (see Figure 7). In this condition, voluntary orienting of attention delayed IOR development. On the other hand, when the endogenous cue was invalid (meaning that IOR was developing in a location opposite to the focus of endogenous attention) IOR was large and highly significant $(F(1,22)=$ $24.622, p<.0001)$, and there was no interaction with SOA (see Figure 8).

\section{Discussion}

Experiment 2 shed some light on the question raised by the previous experiment. When the presentation was binocular, the interaction between the endogenous cue and IOR was difficult to interpret. The monocular presentation enabled us to dissect the different conditions and understand the way these two orienting mechanisms operate. In the temporal hemifield, a constant IOR of $11 \mathrm{msec}$ was found, independent of endogenous orienting of attention. In this hemifield, endogenous attention did not affect IOR at all. In the nasal hemifield, if the endogenous inhibition developed in the same location as IOR (endogenous invalid trials), a strong and constant IOR of $20 \mathrm{msec}$ was found. However, in this hemifield, if the endogenous facilitation developed at the same location as IOR and counteracted it (endogenous valid trials), IOR developed only after an SOA of $700 \mathrm{msec}$.

In daily life, vision is binocular rather than monocular. In this situation, as was shown in Experiment 1, IOR cannot be prevented from developing. A peripheral-visual stimulus elicits IOR in a ballistic and autonomous fashion. In Experiment 1, what appeared to be a smaller IOR when the endogenous cue was valid, and a larger IOR when the endogenous cue was invalid, was in fact the final result from the combination of: (1) the IOR developing through the temporal hemifield of the ipsilateral eye; (2) the IOR developing through the nasal hemifield of the contralateral eye; and (3) the endogenous attention affecting the first but not the second.

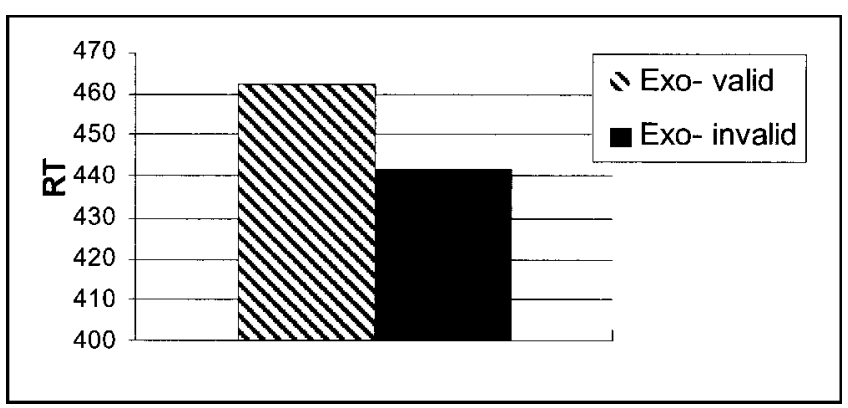

Figure 8. Experiment 2. Nasal hemifield. IOR in the endogenous invalid condition. 


\section{GENERAL DISCUSSION}

In order to examine the endogenous influence on IOR, we presented the endogenous cue after the presumed initiation of IOR. At the beginning of each trial, attention was summoned by an exogenous cue, then, after a certain period of time, the endogenous cue appeared and, lastly, after an additional delay, the target appeared. The validity of each cue was manipulated orthogonally. In Experiment 2, we employed the same paradigm, but used monocular viewing. In this experiment, the endogenous effect on IOR was tested separately in the temporal and nasal hemifields.

The results of this study present additional supporting evidence that the extrageniculate visual pathway, probably the SC, is the neuroanatomic base of the IOR mechanism. The temporal-nasal asymmetry that characterizes this pathway is found also in the modulation of IOR. According to the results of this study, IOR modulation is possible only in the nasal hemifield.

Our motivation for studying the flexibility of IOR was the belief that it could contribute not only to the understanding of this phenomenon itself, but also that it could have implications for the modulation of automatic processes by higher order ones and the monitoring of lower structures by higher cortical processes. The results described above indicate that the IOR mechanism is highly automatic and reflexive. It seems that whenever the right conditions activate the SC (meaning a stimulus appears at the temporal-visual field of the ipsilateral eye), the SC triggers IOR, autonomously and ballistically. ${ }^{5}$ IOR cannot be prevented from developing, even by endogenous-controlled focusing of attention at the location where IOR is developing and will eventually emerge. On the other hand, there seems to be some flexibility in the function of the organism as a unit, since the voluntary focusing of attention can attenuate and delay IOR expression, at least in some situations.

The picture that emerges from this study is that flexibility of the organism in this case might be obtained not by making the automatic procedures themselves flexible, but rather by using them within higher processes, modulating them by cortical structures. In support of this idea, there is growing evidence in the literature that IOR feeds higher processes and interacts with higher processes such as object-based perception (Tipper, Driver, \& Weaver, 1991).

\section{METHODS}

\section{Experiment 1}

\section{Apparatus and Stimuli Display}

An Olivetti M250 personal computer controlled the presentation of the stimuli and the acquisition of data throughout the experiment. Subjects were seated in a dimly illuminated and silent room, and viewed a VGA display monitor with white stimuli on a black background from a distance of $54 \mathrm{~cm}$. Their head rested loosely on a chin rest. The index finger of the preferred hand rested on a response key placed on the table between the subject and the display.

Monitoring of eye movements was done through a small, highly sensitive Pulnix video camera connected to a monitor that was watched by the experimenter throughout the experiment.

The constant display consisted of three $2^{\circ}$ boxes: one box at the center of the screen and two peripheral boxes $10^{\circ}$ from fixation on the left and right sides. At the beginning of each trial, subjects fixated on a dot, which appeared in the middle of the central box. The endogenous cue consisted of a $1.2^{\circ}$ arrow that appeared inside the central box and pointed to the left or to the right. In $80 \%$ of the trials, the arrow pointed to the side where the target appeared, and in the remaining 20\%, the arrow pointed to the opposite side. The exogenous cue consisted of the brightening of one of the boxes. The brightening appeared in $50 \%$ of the trials at each peripheral box. The target stimulus was a $1^{\circ}$ asterisk that appeared inside one of the peripheral boxes ( $50 \%$ of the trials in each box). Additional catch trials were added, creating experimental blocks composed of $20 \%$ empty trials and $80 \%$ target trials.

\section{Procedure}

Each trial consisted of the following sequence (see Figure 1). First, a fixation dot appeared within the central box for $500 \mathrm{msec}$. After $200 \mathrm{msec}$ (i.e., 700 msec since fixation onset), the exogenous cue was presented for $200 \mathrm{msec}$. Then, after a variable interval dependent on the box-arrow SOA (BA-SOA), the endogenous cue was presented for another $200 \mathrm{msec}$ (in Experiment 2, the interval between the exogenous cue and the endogenous cue was fixed). Finally, after a variable arrow-target SOA (AT-SOA), the target appeared until the subject's response, but no more than for $1500 \mathrm{msec}$.

The interval between the endogenous cue onset and the target onset (AT-SOA) could be 150, 300 or 500 msec. The interval between the exogenous cue onset and the endogenous cue onset (BA-SOA) could be 200, 400 or $600 \mathrm{msec}$ (as mentioned, this interval was manipulated only in Experiment 1, and it was fixed in Experiment 2). It is important to emphasize that all the SOAs between the exogenous cue and the target were long enough to produce IOR. For example, the shortest SOA was $350 \mathrm{msec}$, composed of BA-SOA of 200 msec and AT-SOA of 150 msec.

The next trial began after an intertrial interval of 1500 msec.

Simple RT was measured in msec. Responses shorter than $100 \mathrm{msec}$ were treated as guesses; in which case, a "too soon" message was presented, the response was 
discarded and the trial was presented again. Responses longer than $1500 \mathrm{msec}$ were not included in the analysis.

Each subject was tested in a 1-hr session. The session started with 40 trials of practice. Subjects were instructed about cue validities and were encouraged to keep fixation, pay attention to the endogenous cue, ignore the exogenous cue, and to respond as fast as possible, but not before the target appeared. During practice, the subjects received feedback from the experimenter about their success in avoiding eye movements.

\section{Design}

The experimental design was factorial, with four within-subjects independent variables (endogenous cue validity, exogenous cue validity, AT-SOA, BA-SOA). A total of 1080 trials were presented during a session. The target appeared in 900 of them and 180 were catch trials. From the 900 nonempty trials: (i) for the endogenous cue, 720 trials were valid $(9$ combinations of the timing variables $\times 2$ sides $\times 40$ repetitions) and 180 trials were invalid ( 9 combinations of the timing variables $\times 2$ boxes $\times 10$ repetitions); and (ii) for the exogenous cue, 450 trials were valid (9 combinations of the timing variables $\times 2$ boxes $\times 25$ repetitions) and 450 were invalid (according to the same distribution). The various trial types were presented randomly during the session.

\section{Subjects}

Sixteen undergraduates from the Behavioral Sciences Department at Ben Gurion University of the Negev participated in the experiment in partial fulfillment of a course requirement. They all had normal, or corrected to normal, vision. The results of one of the subjects were omitted from the analysis since she could not prevent eye movements.

\section{Experiment 2}

\section{Apparatus and Stimuli Display}

Apparatus and stimuli were the same as in Experiment 1. The main difference was that subjects wore an eyepatch, covering one of their eyes. Each subject was tested in two blocks, one block with each eye (half of the subjects were tested first with their right eye and then with their left eye, and half of the subjects were tested in the opposite order). In the monocular setting, for the right eye, the right side of the monitor was the temporal hemifield and the left side of the monitor was the nasal hemifield. For the left eye, the right side of the monitor was the nasal hemifield and the left side of the monitor was the temporal hemifield.

\section{Design}

The experimental design was factorial, with four withinsubjects independent variables (endogenous cue validity, exogenous cue validity, SOA, hemifield). Since the BA-SOA variable was found to be not significant in the previous experiment, in Experiment 2 we decided to use only one fixed interval between the box and the arrow cues. This interval was set to $200 \mathrm{msec}$. The SOA between the endogenous cue onset and the target onset could be 150, 300 or $500 \mathrm{msec}$ (meaning 350, 500, and 700 exogenous cue-target SOA).

In each block there were 462 trials. The target appeared in 420 of them (90.9\%), and 42 were catch trials. From the 420 nonempty trials: (i) for the endogenous cue, 336 trials were valid ( 3 SOAs $\times 2$ boxes $\times 56$ repetitions) and 84 trials were invalid ( 3 SOAs $\times 2$ boxes $\times 14$ repetitions); (ii) for the exogenous cue, 210 trials were valid (3 SOAs $\times 2$ boxes $\times 35$ repetitions) and 210 were invalid (according to the same distribution). The various trial types were presented randomly.

\section{Subjects}

Twenty-four undergraduates from the Behavioral Sciences Department at Ben Gurion University of the Negev participated in the experiment in partial fulfillment of a course requirement. They all had normal, or corrected to normal, vision. The results of one of the subjects were omitted from the analysis since she could not prevent eye movements.

\section{Acknowledgments}

This research was supported by the Ben-Gurion Ph.D. Fellowship, the Israeli Ministry of Sciences and Art, and by the Israel Foundations Trustees Ph.D. Fellowship. Both fellowships were given to the first author. The first author also belongs to the Sackler Institute for Human Brain Development, Weill Medical College, Cornell University, New York.

The authors wish to thank the reviewers for their suggestions and helpful corrections regarding the previous version of this manuscript.

Reprint requests should be sent to: Dr. Andrea Berger, Behavioral Sciences Dept., Ben-Gurion University of the Negev, Beer-Sheva 84105, Israel; email: andrea@bgumail.bgu.il.

\section{Notes}

1. Unless the endogenous cue serves as a trigger for preparing a saccade (Schmidt, 1996; Rafal et al., 1989).

2. AT-SOA was the interval between the beginning of the endogenous precue and the beginning of the target. For more details, see the Methods section.

3. BA-SOA was the interval between the beginning of the exogenous precue and the beginning of the endogenous precue. For more details, see the Methods section.

4. In addition, there were two less-interesting main effects: BASOA effect showing slightly shorter RTs when its value was 400 msec (mean RTs were 411, 404, and $414 \mathrm{msec}$ in the 200-, 400-, 
and 600-msec intervals, respectively), $(F(2,28)=7.84, M S E=$ $622.5, p<.01$ ); and AT-SOA main effect (mean RTs were 426, 400 , and $403 \mathrm{msec}$ in the 150-, 300-, and 500-msec intervals, respectively), $(F(2,28)=20.2, M S E=1,824, p<.0001)$.

5. These are currently considered to be the main criteria when defining automatic processes (Bargh, 1992; Tzelgov, 1997).

\section{REFERENCES}

Bargh, J. A. (1992). The ecology of automaticity: Towards establishing the conditions needed to produce automatic processing effect. American Journal of Psychology, 105, 181-199.

Berger, A., Dori, H., \& Henik, A. (1999). Peripheral non-informative cues do induce early facilitation of target detection. European Journal of Cognitive Psychology, 11, 119-137.

Berger, A., Henik, A., Rafal, R., \& Egly, R. (submitted). Competition between endogenous and exogenous orienting of visual attention.

Briand, K. A., \& Klein, R. M. (1987). Is Posner's "beam" the same as Triesman's "glue"?: On the relation between visual orienting and feature integration theory. Journal of Experimental Psychology: Human Perception and Performance, 13, 228-241.

Danziger, S., Fendrich, R., \& Rafal, R. D. (1997). Inhibitory tagging of locations in the blind field of hemianopic patients. Consciousness and Cognition, 6, 291-307.

Hubel, D. H., LeVay, S., \& Wiesel, T. N. (1975). Mode of termination of retino-tectal fibers in macaque monkey: An autoradiographic study. Brain Research, 96, 25-40.

Jonides, J. (1981). Voluntary versus automatic control over the mind's eye's movement. In J. B. Long \& A. D. Baddeley (Eds.), Attention and performance IX (pp. 187-203). Hillsdale, NJ: Erlbaum.

Kwak, H. (1996). Can inbibition of return be inbibited by endogenous orienting? Paper presented at the 37th Annual Meeting of the Psychonomic Society, Chicago.

Mauer, D., \& Lewis, T. L. (1998). Overt orienting toward peripheral stimuli: Normal development and underlying mechanisms. In J. E. Richards (Ed.), Cognitive neuroscience of attention: A developmental perspective (pp. 51-102). Hillsdale, NJ: Erlbaum.

Maylor, E. A. (1985). Facilitory and inhibitory components of orienting in visual space. In M. I. Posner \& O. S. M. Marin (Eds.), Attention and performance XI (pp. 189-204). Hillsdale, NJ: Erlbaum.

Maylor, E. A., \& Hockey, R. (1985). Inhibitory component of externally controlled covert orienting in visual space. Journal of Experimental Psychology: Human Perception and Performance, 11, 777-787.

Maylor, E. A., \& Hockey, R. (1987). Effects of repetition on the facilitory and inhibitory components of orienting in visual space. Neuropsychologia, 25, 41-55.

Muller, H. J., \& Humphreys, G. W. (1991). Luminance increment detection: Capacity-limited or not? Journal of Experimental Psychology: Human Perception and Performance, 17, 107-124.

Pollack, J. G., \& Hickey, T. L. (1979). The distribution of retino- collicular axon terminals in rhesus monkey. Journal of Comparative Neurology, 185, 587-602.

Posner, M. I. (1980). Orienting of attention. Quarterly Journal of Experimental Psychology, 32, 3-25.

Posner, M. I., \& Cohen, Y. P. C. (1980). Attention and the control of movements. In G. E. Stelmach \& J. Requin (Eds.), Tutorials in motor behavior (pp. 243-258). Amsterdam, the Netherland: North Holland.

Posner, M. I., \& Cohen, Y. P. C. (1984). Components of visual orienting. In H. Bouma \& D. Bouwhuis (Eds.), Attention and performance $X$ (pp. 531-556). London, England: Erlbaum.

Posner, M. I., Rafal, R. D., Choate, L., \& Vaughn, J. (1985). Inhibition of return: Neural basis and function. Cognitive Neuropsychology, 2, 211-228.

Rafal, R., Calabresi, P., Brennan, C., \& Sciolto, T. (1989). Saccade preparation inhibits reorienting to recently attended locations. Journal of Experimental Psychology: Human Perception and Performance, 15, 673-685.

Rafal, R., \& Henik, A. (1994). Inhibitory neural mechanisms for integrating controlled and automatic processes. In D. Dagenbach \& T. H. Carr (Eds.), Inhibitory processes in attention, memory and language (pp. 1-51). San Diego, CA: Academic Press.

Reuter-Lorenz, P. A., \& Frendrich, R. (1992). Oculomotor readiness and covert orienting: Differences between central and peripheral precues. Perception and Psychophysics, 52, 336-344.

Sapir, A., Soroker, N., Berger, A., \& Henik, A. (1999). "Inhibition of return"' in spatial attention: Direct evidence for collicular generation. Nature Neuroscience, 2, 1053-1054.

Schmidt, W. C. (1996). "Inhibition of return" without visual input. Neuropsychologia, 34, 943-952.

Sherman, S. M. (1974). Visual fields of cats with cortical and tectal lesions. Science, 185, 355-357.

Simion, F., Valenza, E., Umilta, C., \& Dalla-Baba, B. (1995). Inhibition of return in newborns is temporo-nasal asymetrical. Infant Behavior and Development, 18, 189194.

Taylor, T. L., \& Klein, R. M. (1998). On the causes and effects of inhibition of return. Psychonomic Bulletin and Review, 5, 625-643.

Tipper, S. P., Driver, J., \& Weaver, B. (1991). Object-centred inhibition of return of visual attention. Quarterly Journal of Experimental Psychology, 43A, 289-98.

Tzelgov, J. (1997). Specifying the relations between automaticity and consciousness: A theoretical note. Consciousness and Cognition, 6, 441-451.

Williams, C., Azzopardi, P., \& Cowey, A. (1995). Nasal and temporal retinal ganglion cells projecting to the midbrain: Implications for "blindsight". Neuroscience, 65 , 577-586.

Wilson, M. E., \& Toyne, M. G. (1970). Retino-tectal and corticotectal projections in Macaca mulatta.Brain Research, 24, 395-406.

Yantis, S., \& Jonides, J. (1990). Abrupt visual onsets and selective attention: Voluntary versus automatic allocation. Journal of Experimental Psychology: Human Perception and Performance, 16, 121-134. 